\title{
Nonlinearity investigation of reliability-based topology optimization strategies with application to total hip replacement
}

\author{
Ghais Kharmanda ${ }^{1,{ }^{*}}$, Hasan Mulki ${ }^{2}$, and Imad Antypas $2^{3}$ \\ ${ }^{1}$ INSA Rouen, 685 Avenue de l'Université, 76800 Saint-Étienne-du-Rouvray, France \\ ${ }^{2}$ College of Engineering and Technology, American University of the Middle East, Kuwait \\ ${ }^{3}$ Don State Technical University, 1, Gagarin Sq., 344003 Rostov-on-Don, Russia
}

\begin{abstract}
In literature, the topology optimization can be divided into two main models. The first model is called Deterministic Topology Optimization (DTO) producing a single configuration for a given design space. The second one is called Reliability-Based Topology Optimization (RBTO) generating several layouts. In our previous work, two approaches considering the concept of Inverse Optimum Safety Factors (IOSF) were elaborated and only applied to the normal distribution being linear distribution. In this work, a nonlinearity investigation is presented to compare between the linear and nonlinear distribution. The RBTO developments are applied to the total hip replacement to provide suitable hollow stems at the conceptual design stage. The nonlinearity presented here, is related to the types of the random variable distributions. The most common distributions such as normal, lognormal, uniform and Weibull are considered here to perform the investigation. The results show the nonlinearity effect on the output parameter values, but lead to almost similar layouts of the resulting hollow stems in several cases. In certain types of distributions such as Weibull, the changes on the input parameters are very variant. At certain values of the reliability index, some input parameters of material properties exceeded their limits and the algorithm stopped.
\end{abstract}

\section{Introduction}

In the Deterministic Design Optimization, the solutions are obtained without taking uncertainty into account, that may lead to unreliable designs. the Reliability-Based Design Optimization (RBDO) seeks to find the structures that should be reliable and economic by considering the uncertainty during the detailed design stage. However, to integrate the uncertainty at the conceptual design stage, the topology optimization should be introduced considering a different integration philosophy since the topology optimization is quantitative. Thus, the integration of uncertainty is carried out in a different way and then lead to different findings.

\footnotetext{
* Corresponding author: imad.antypas@mail.ru
} 
Before 2000, one of the main challenges in the different topology optimization studies was to obtain the unique solution for a given design space. Sometimes the resulting unique solution is prone to manufacturability problems. However, a Reliability-Based Topology Optimization (RBTO) model has been developed in 2001 [1] to generate several solutions with different advantages. It allows us to select the best solution to fulfill the technical specifications. The structural weight of the resulting topologies obtained by this model is reduced when comparing with the DTO model. Furthermore, when the RBTO model leads to more reliable structures compared to the DTO model for the same weight levels (The interested reader can refer to [2] to see several kinds of applications). Two points of view are presented for the RBTO developments: topology optimization and reliability analysis (For detailed comparison, the interested reader can refer to [3-5]). Considering the previous works, it has been shown that the point of view 'topology optimization' seems to be interesting for structural designers since it provides several reliability-based structures relative the reliability index changes. It leads to different layout structures while the developments from a point of view 'reliability analysis' leads to same configurations with different densities that have no sense for integrating the reliability concept.

To perform the RBTO problems, several RBDO approaches can be adopted since we deal with different philosophies. The RBDO methods have been already developed regarding to their use [2]. For example, the OSF method has been simply implemented by performing two main procedures. The first one is to find the failure point performing a simple optimization loop while the second one consists of using the Optimum Safety Factor (OSF) formulations to compute the optimum solution. In this work, the OSF method is adopted in another way for the RBTO model, the developed Inverse Optimum Safety (IOSF) method consists of first reaching the configuration using Deterministic Topology Optimization (DTO). Next the OSF formulations are used with inverse derivative signs in order to generate several reliabilitybased topologies. The integration of the DTO and RBTO into hip prosthesis stems is motivated as a practical case. These models are considered as effective tools for the conceptual design stage to delete un-needed structural region and also to generate transversal holes allowing a good fixation level after the healing period.

\section{Materials and Methods}

\subsection{Reliability Index}

In order to produce different topologies, a reliability index $\beta$ is used with a normalized vector u. Fig. 1 shows the transformation image between the random variables $\mathbf{y}$ in the physical space and the normalized vector $\mathbf{u}$ in the normalized space [6-10]. The reliability index assessment is carried out using FORM (First Order Reliability Method). The evaluation of the reliability index can be performed using an optimization procedure:

$$
\beta=\operatorname{mind}(u) \text { s.t.: } H(u)=0
$$

where the distance $d(u)$ in the normalized space is given by:

$$
d(u)=\sqrt{\sum_{i=1}^{n} u_{i}^{2}}
$$

This procedure seeks to find the optimum value of $\beta$ which corresponds to the Most Probable failure Point (MPP). This point is represented by the minimum distance between 
the origin of the normalized space and limit state curve as shown in Fig. 1. In structural engineering, the reliability index interval has to be: $\beta \in[3-4.25]$.

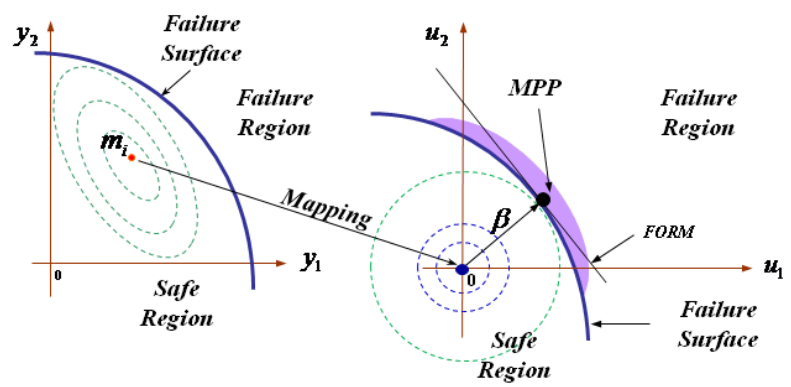

Fig. 1. Transformation image between the physical space and the normalized one

\subsection{DTO versus RBTO}

The resulting topologies are obtained considering two different approaches: Objective-Based Approach (OBA) and Performance-Based Approach (PBA).

\subsubsection{Objective-Based Approach (OBA)}

In this case, the objective is to minimize the structural compliance respecting a target ratio decrease of volume $V_{f}^{\text {Tar }}$ [11-16]. The problem of DTO model is then mathematically described by:

$$
\begin{gathered}
\min : \operatorname{Com}(x) \\
\text { s.t.: } \frac{\operatorname{Vol}(x)}{V^{0}} \leq V_{f}^{\mathrm{Tar}}
\end{gathered}
$$

where $\operatorname{Com}(x)$ is the structural compliance considering the densities of the used material in each element as optimization variables. Their values should be found in the interval $[0,1]$. $V^{0}$ and $\operatorname{Vol}(x)$ are the initial- and current structural volume values. When integrating the reliability constraint, the RBTO problem is then mathematically written as:

$$
\begin{gathered}
\min : \operatorname{Com}(x) \\
\text { s.t.: } \beta \geq \beta_{t} \\
\text { and: } \frac{\operatorname{Vol}(x)}{V^{0}} \leq V_{f}^{\text {Tar }}
\end{gathered}
$$

where $\beta_{t}$ is the required reliability index to be satisfied. The optimum value of the normalized vector can be written by:

$$
u_{i}= \pm \beta_{t} \sqrt{\frac{\left|\frac{\partial F}{\partial y_{i}}\right|}{\sum_{j=1}^{n}\left|\frac{\partial F}{\partial y_{j}}\right|}}
$$


Considering the derivative sign of the objective function with respect to random variables $y_{i}$, we write:

$$
\frac{\partial F}{\partial y_{i}}>0 \Leftrightarrow u_{i}>0 \text { and } \frac{\partial F}{\partial y_{i}}<0 \Leftrightarrow u_{i}<0
$$

\subsubsection{Performance-Based Approach (OBA)}

Considering this approach, the objective is to minimize the structural volume under a target ratio increase of compliance $C_{f}^{\mathrm{Tar}}$. The DTO problem is then mathematically written as follows:

$$
\begin{gathered}
\min : \operatorname{Vol}(x) \\
\text { s.t.: } \frac{\operatorname{Com}(x)}{C^{0}} \leq 1+C_{f}^{\mathrm{Tar}}
\end{gathered}
$$

where $\operatorname{Vol}(x)$ is the structural volume considering the material densities in each element as optimization variables and belong to the interval $[0,1] . C^{0}$ and $\operatorname{Com}(x)$ represent the initialand current structural compliance values. When integrating the reliability constraint, the RBTO problem is then mathematically written as:

$$
\begin{gathered}
\min : \operatorname{Vol}(x) \\
\text { s.t.: } \beta \geq \beta_{t} \\
\text { and: } \frac{\operatorname{Com}(x)}{C^{0}} \leq 1+C_{f}^{\mathrm{Tar}}
\end{gathered}
$$

The optimum value of the normalized vector can be expressed by:

$$
u_{i}= \pm \beta_{t} \sqrt{\frac{\left|\frac{\partial G}{\partial y_{i}}\right|}{\sum_{j=1}^{n}\left|\frac{\partial G}{\partial y_{j}}\right|}}
$$

According to the derivative sign of the limit state function with respect to random variables $y_{i}$, we write:

$$
\frac{\partial G}{\partial y_{i}}>0 \Leftrightarrow u_{i}>0 \text { and } \frac{\partial G}{\partial y_{i}}<0 \Leftrightarrow u_{i}<0
$$

In both developed approaches, it is considered that the starting point represents the failure point $P_{y}$ and next the reliability-based topology $P_{x}$ is found. The reliability-based topology $P_{x}$ must be more reliable than the failure point $P_{y}$ and must also verify a required reliability index $\beta_{t}$. This way the DTO procedure is used to find the failure point $P_{y}$ and the both developed approaches are then used to determine the reliability-based topologies $P_{x}$. The OSF formulations for the normal, lognormal, uniform and Weibull distributions can be found in [2]. 


\subsection{Total Hip Replacement}

The RBTO can be integrated at the conceptual design stage to sketch several types of hollow stems according to the daily loading cases [17-19]. In this section, a 2D model [19] is considered for simplicity to carry out the different topology optimization studies. Figure 2a shows the studied 2D stem model implanted in the cortical and cancellous bone tissues and also the applied body and muscle forces $\left(F^{B}\right.$ and $\left.F^{M}\right)$. Their different component values in two directions $\left(F_{x}^{B}, F_{y}^{B}, F_{x}^{M}\right.$ and $\left.F_{y}^{M}\right)$ are found in Table 1 [17-19]. Figure 2b shows the different areas to be specified as optimized or non-optimized domains. The inner area of the shaft $A_{1}$ is assumed to be the optimized domain, while the other areas to be non-optimized domains: $A_{2}$ contains the head, neck, shoulder and an outer area of the shaft, $A_{3}$ contains the cortical tissue and $A_{4}$ contains the cancellous tissue.
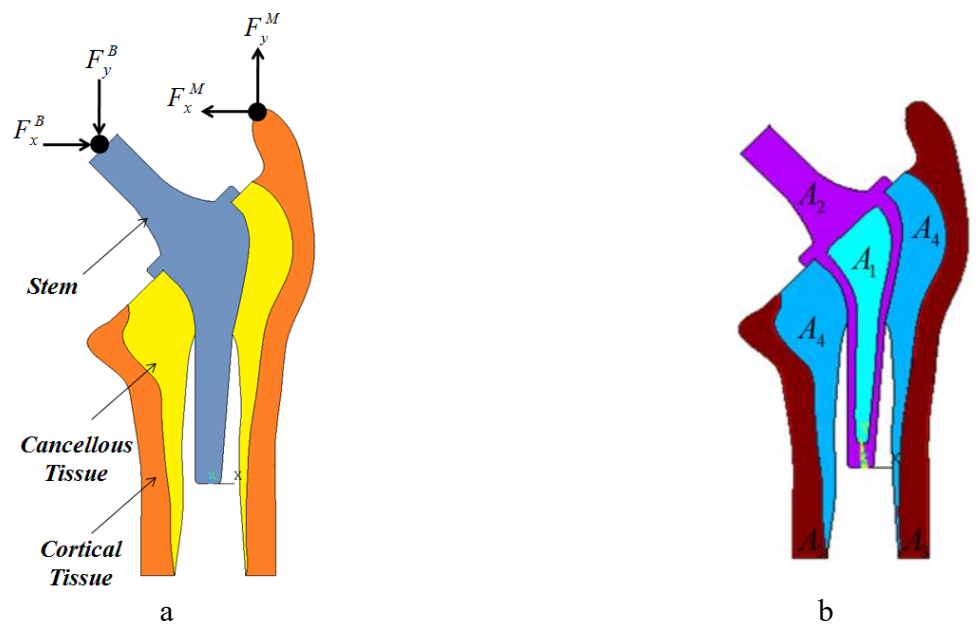

Fig. 2: a) studied 2D stem model implanted in bone tissues with applied loads and b) Inner area as an optimized domain

Table 1. Applied body and muscle forces [17-19]

\begin{tabular}{|c|c|c|c|}
\hline \multicolumn{2}{|c|}{ Loading Cases } & $\boldsymbol{F}_{\boldsymbol{x}}[\mathbf{N}]$ & $\boldsymbol{F}_{\boldsymbol{y}}[\mathbf{N}]$ \\
\hline \multirow{2}{*}{$L 1$} & $F^{B}$ & 942 & 2117 \\
\cline { 2 - 4 } & $F^{M}$ & 330 & 621 \\
\hline \multirow{2}{*}{$L 2$} & $F^{B}$ & -2997 & 1119 \\
\cline { 2 - 4 } & $F^{M}$ & -49 & 348 \\
\hline \multirow{2}{*}{$L 3$} & $F^{B}$ & 1283 & 866 \\
\cline { 2 - 4 } & $F^{M}$ & 268 & 383 \\
\hline
\end{tabular}

For 2D and 3D models, all used materials are considered homogeneous and linear elastic. The cortical bone tissue is assumed to be isotropic with Young's modulus $E=17 \mathrm{GPa}$ and Poisson's ratio $v=0.33$. The cancellous bone tissue is also assumed to be isotropic material with Young's modulus $E=386 \mathrm{MPa}$ and Poisson's ratio $v=0.33$. The stem is made of titanium alloy with Young's modulus $E=110 \mathrm{GPa}$ and Poisson's ratio: $v=0.3$ [17-19]. 


\section{Results and Discussion}

In this section, the different results and treated and discussed at two levels. The first level concerns the numerical values of the compliance and the volume supported by curves representing the changes of the compliance and the volume in function of reliability index values. The second level concerns the resulting layouts for both approaches and considering the values of reliability index.

Table 2 represents the numerical DTO and RBTO results for Objective-Based Approach (OBA). When considering Weibull distribution, the change leads to exceed the limit of the Poisson's ratio at $\beta=4.25$ for $L 2$. This way the topology optimization cannot be performed.

Table 2. Numerical DTO and RBTO results for Objective-Based Approach (OBA)

\begin{tabular}{|c|c|c|c|c|c|c|c|}
\hline \multirow[b]{2}{*}{ Type } & \multirow[b]{2}{*}{$\beta$} & \multicolumn{2}{|c|}{$L 1$} & \multicolumn{2}{|c|}{$L 2$} & \multicolumn{2}{|c|}{$L 3$} \\
\hline & & Com & Vol & Com & Vol & Com & Vol \\
\hline DTO-OBA & 0 & 0.74 & 351.37 & 0.72 & 351.38 & 0.37 & 351.38 \\
\hline \multirow{3}{*}{$\begin{array}{c}\text { RBTO-OBA } \\
\text { Normal }\end{array}$} & 3 & 0.95 & 321.77 & 0.85 & 383.62 & 0.77 & 307.89 \\
\hline & 3.8 & 1.01 & 313.88 & 0.91 & 392.22 & 0.91 & 296.3 \\
\hline & 4.25 & 1.05 & 309.44 & 0.93 & 397.06 & 1.01 & 289.78 \\
\hline \multirow{3}{*}{$\begin{array}{l}\text { RBTO-OBA } \\
\text { (Lognormal) }\end{array}$} & 3 & 0.74 & 351.38 & 0.87 & 380.51 & 0.8 & 303.22 \\
\hline & 3.8 & 1.3 & 309.97 & 0.94 & 388.29 & 0.99 & 289.85 \\
\hline & 4.25 & 1.06 & 304.99 & 0.96 & 392.57 & 1.11 & 282.11 \\
\hline \multirow{3}{*}{$\begin{array}{c}\text { RBTO-OBA } \\
\text { (Uniform) }\end{array}$} & 3 & 1.04 & 310.6 & 0.93 & 386.5 & 0.8 & 295.15 \\
\hline & 3.8 & 1.1 & 301.8 & 0.96 & 392.54 & 0.85 & 287.93 \\
\hline & 4.25 & 1.15 & 297.53 & 0.97 & 394.44 & 0.86 & 284.73 \\
\hline \multirow{3}{*}{$\begin{array}{c}\text { RBTO-OBA } \\
\text { (Weibull) }\end{array}$} & 3 & 0.95 & 322.29 & 0.91 & 380.06 & 0.93 & 301.2 \\
\hline & 3.8 & 1.03 & 310.84 & - & - & 1.35 & 279.95 \\
\hline & 4.25 & 1.07 & 303.77 & - & - & 1.68 & 266.25 \\
\hline
\end{tabular}

Table 3 represents the numerical DTO and RBTO results for Performance-Based Approach (PBA). When considering Weibull distribution, the change leads to exceed the limit of the Poisson's ratio at $\beta=4.25$ for $L 2$. This way the topology optimization cannot be performed.

Table 3. Numerical DTO and RBTO results for Performance-Based Approach (PBA)

\begin{tabular}{|c|c|c|c|c|c|c|c|}
\hline \multirow{2}{*}{ Type } & \multirow{2}{*}{$\beta$} & \multicolumn{2}{|c|}{ L1 } & \multicolumn{2}{c|}{ L2 } & \multicolumn{2}{c|}{ L3 } \\
\cline { 3 - 8 } & & Com & Vol & Com & Vol & Com & Vol \\
\hline DTO-PBA & 0 & 1.07 & 149.66 & 0.95 & 107.22 & 0.48 & 264.7 \\
\hline \multirow{2}{*}{$\begin{array}{c}\text { RBTO-PBA } \\
\text { Normal }\end{array}$} & 3 & 1.97 & 89.95 & 1.04 & 132.39 & 0.94 & 253.94 \\
\cline { 2 - 8 } & 4.8 & 2.28 & 85.03 & 1.07 & 134.24 & 1.1 & 249.72 \\
\hline RBTO-PBA & 3 & 2.45 & 85.85 & 1.08 & 134.12 & 1.19 & 246.3 \\
\hline
\end{tabular}




\begin{tabular}{|c|c|c|c|c|c|c|c|}
\hline \multirow[t]{2}{*}{ (Lognormal) } & 3.8 & 2.45 & 83.71 & 1.09 & 133.43 & 1.17 & 246.84 \\
\hline & 4.25 & 2.69 & 85.08 & 1.1 & 134 & 1.29 & 243.86 \\
\hline \multirow{3}{*}{$\begin{array}{c}\text { RBTO-OBA } \\
\text { (Uniform) }\end{array}$} & 3 & 1.97 & 89.5 & 1.09 & 133.28 & 0.95 & 250.03 \\
\hline & 3.8 & 2.03 & 88.62 & 1.12 & 132.99 & 0.99 & 247.58 \\
\hline & 4.25 & 2.05 & 88.36 & 1.14 & 129.34 & 1.01 & 246.35 \\
\hline \multirow{3}{*}{$\begin{array}{c}\text { RBTO-OBA } \\
\text { (Weibull) }\end{array}$} & 3 & 2.43 & 86.38 & 1.04 & 131.78 & 1.13 & 249.89 \\
\hline & 3.8 & 3.33 & 85.09 & - & - & 1.55 & 245.48 \\
\hline & 4.25 & 4.05 & 78.84 & - & - & 1.89 & 242.77 \\
\hline
\end{tabular}

Figures 3a, b and c show the modeling of the numerical results in Tables 2 and 3 for $L 1$, $L 2$ and $L 3$, respectively. In this Fig., some abbreviations are considered. For the output parameters, $\mathrm{C}$ and $\mathrm{V}$ refer to the compliance and volume changes, receptively. For the used approach, $\mathrm{O}$ and $\mathrm{P}$ refer to the OBA and PBA, respectively. For the distribution law, N, L, U and $\mathrm{W}$ refer to Normal, Lognormal, Uniform and Weibull distributions, respectively.
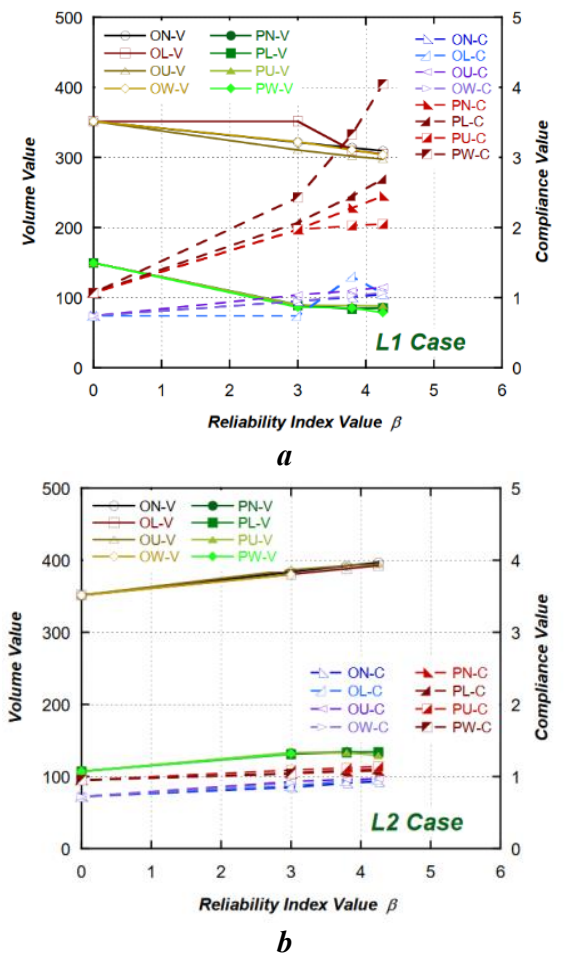


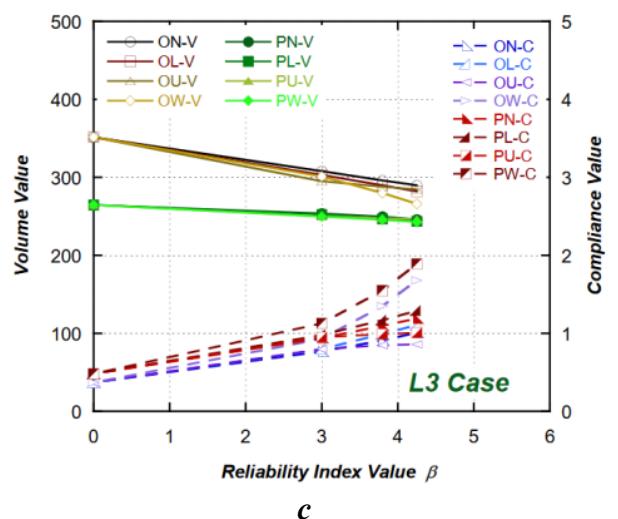

Fig. 3. Modeling of the numerical results for a) $L 1$, b) $L 2$ and c) $L 3$

Figs. 4, 5, 6 and 7 represent different resulting layouts for the daily loading cases: $L 1, L 2$ and $L 3$, respectively. The materials densities represented by several colors where the red color refers to a full material (solid element) and the blue represent a void element (no material). In structural engineering the reliability index values are found in range $\beta \in[3-4.25]$. In this study, three values are selected to investigate the nonlinearity effect of the layout changes: $\beta=3,3.8$ and 4.25. All numerical DTO and RBTO results are presented in Tables 2 and 3 considering both OBA and PBA. The structural compliance and volume values change in function with the reliability index values for the three considered loading cases, however the presented figures correspond to only the layouts changes. Figure 4 shows the resulting DTO layouts [20] for the three loading cases when performing the Objective-Based IOSF Approach.
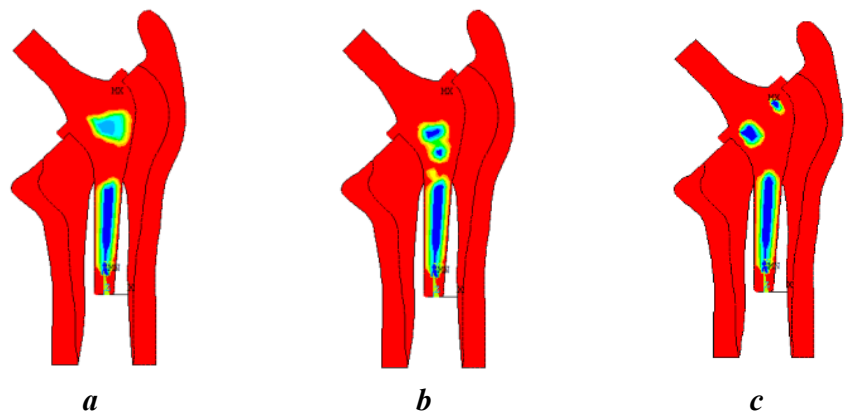

Fig. 4. Resulting DTO layouts for the three daily cases a) $L 1$, b) $L 2$ and c) $L 3$ when performing the Objective-Based IOSF Approach

For the Objective-Based IOSF Approach and for all studied distribution types, when the reliability index values, the different layouts for L1 and L2 are almost similar to the DTO configurations shown in Figures $4 \mathrm{a}$ and c. However, for L2, the resulting RBTO layout for lognormal distribution and $\beta=3$ is similar to DTO layout shown in Figure $4 \mathrm{~b}$. For $\beta=3$, Figures $5 \mathrm{a}, \mathrm{b}, \mathrm{c}$ and $\mathrm{d}$ show the configurations for the normal, lognormal, uniform and Weibull distributions, respectively. For $\beta=3.8$, and $\beta=4.25$, the layouts are almost similar to Figure 5c, except for Weibull distribution where the Poisson's rate become higher than 0.4 and the algorithm is stopped. 


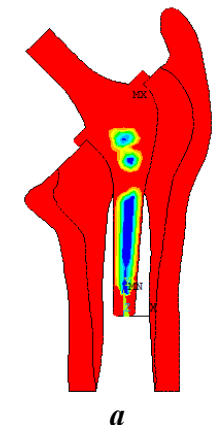

$a$

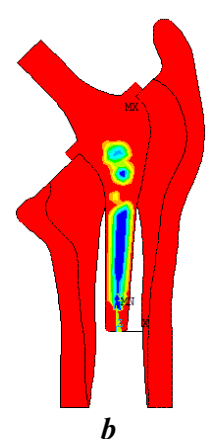

$b$

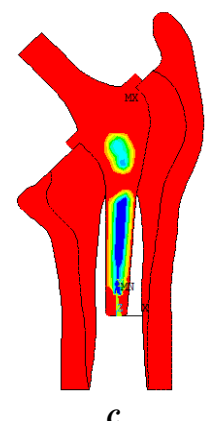

$c$

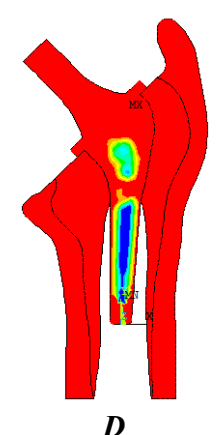

D

Fig. 5. RBTO layouts when using the first approach for a) normal, b) lognormal, c) uniform and d) Weibull distribution for the second loading case $L 2$ and for $\beta=3$.

Figure 6 shows the resulting DTO layouts for the three loading cases when performing the Performance-Based IOSF Approach.

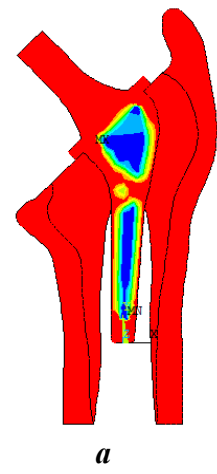

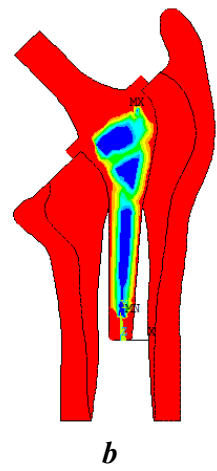

b

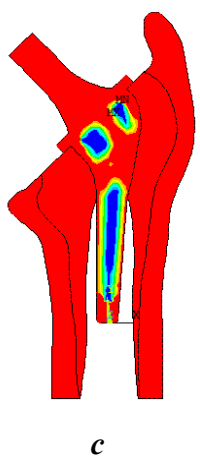

Fig. 6. Resulting DTO layouts for the three daily cases a) $L 1$, b) $L 2$ and c) $L 3$ when performing the Performance-Based IOSF Approach

For $L 1$, Figure 7 a shows the resulting RBTO layout for normal distribution and $\beta=3$. All other layouts are similar to this one for all distributions and all values of reliability index. For L2, the resulting layouts for all distributions and all reliability index values are similar to the resulting DTO configuration shown in Figure $6 \mathrm{~b}$, except for Weibull distribution when $\beta=3.8$, and $\beta=4.25$, where the Poisson's rate become higher than 0.4 and the algorithm stopped. For $L 3$, Figure $7 \mathrm{~b}$ shows the resulting RBTO layout for normal distribution and $\beta=3$. All other layouts are similar to this one for all distributions and for $\beta=3$. Figure $7 \mathrm{c}$ shows the resulting RBTO layout for normal distribution and $\beta=4.25$. For this distribution, the resulting layout for $\beta=3.8$, is almost similar to the resulting layout for uniform distribution illustrated in Figure 7e. Figure 7d shows the resulting RBTO layout for lognormal distribution and $\beta=4.25$. For this distribution, the resulting layout for $\beta=3.8$, is almost similar to the resulting layout for normal distribution illustrated in Figure $7 \mathrm{~b}$. Figure $7 \mathrm{e}$ shows the resulting RBTO layout for uniform distribution and $\beta=4.25$. For this distribution, the resulting layout for $\beta=3.8$, is almost similar to the DTO layout illustrated in Figure 6c. Figure $7 \mathrm{f}$ shows the resulting RBTO layout for Weibull distribution and $\beta=4.25$. For this distribution, the resulting layout for $\beta=3.8$, is almost similar. 


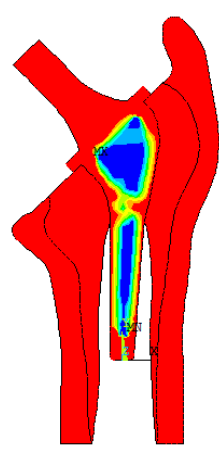

$\boldsymbol{a}$

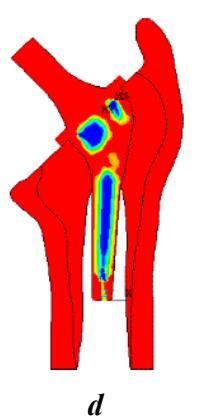

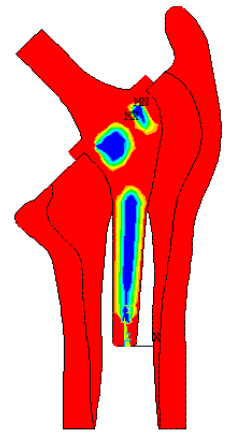

$b$

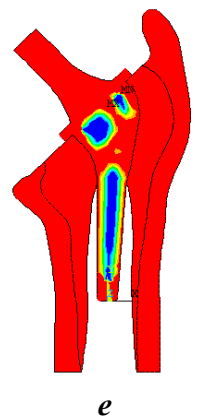

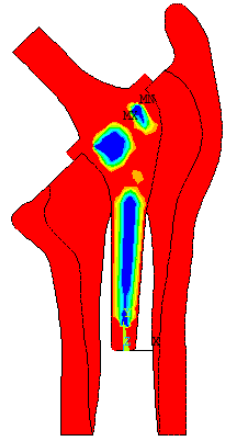

$\boldsymbol{c}$

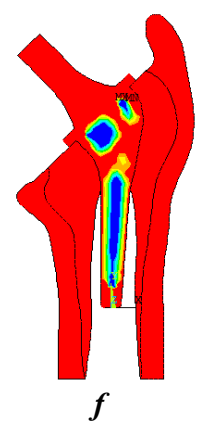

Fig. 7. RBTO-PBA layouts for a) $L 1$, normal distribution and $\beta=3$, b) $L 3$, normal distribution and $\beta=3$, c) $L 3$, normal distribution and $\beta=4.25$, d) $L 3$, lognormal distribution and $\beta=4.25$, e) $L 3$, uniform distribution and $\beta=4.25$, and f) $L 3$, Weibull distribution and $\beta=4.25$.

The resulting topologies can be changed when considering several distributions considering certain loading cases. For the studied cases, there is no big change in the layouts, that maybe because the number of input parameters is small. To identify the effect, the geometrical parameters should be included. According to the numerical results, it can be noted that despite the uniform distribution is not linear, the corresponding results (compliance) are less sensitive to the change of reliability index values. However, the highest changes are noted when using Weibull distribution which is the most used distribution in reliability studies. It indicates highest increase of compliance with almost little change in volume, which shows disadvantage to provide a rigid and light structure. While the uniform distribution leads in certain cases to a small increase in compliance with relatively good reduction in volume for most of loading cases.

\section{Conclusions}

The integration of the RBTO is carried out into the total hip replacement in order to provide several kinds of hollow stems with object of increasing the prosthesis performance, especially osseointegration. The uncertainty is applied on the material properties, loads and constraint boundaries. Two RBTO approaches are developed here and lead in certain cases to different layouts compared to the DTO configurations. It is very important to consider the uncertainty on the stem geometry in order to generate new layouts with significant difference. An investigation of nonlinearity is carried out considering the most common distribution in this field. A significant change in the output parameter can be observed when selecting the Weibull distribution law. 


\section{References}

1. G. Kharmanda, N. Olhoff, Reliability-Based Topology Optimization, Report $\mathrm{N}^{\circ}: 110$, Institute of Mechanical Engineering, Aalborg University, Denmark, December (2010)

2. G. Kharmanda, A. El-Hami, Biomechanics: Optimization, Uncertainties and Reliability, ISTE \& Wiley, ISBN: 9781786300256, January (2017)

3. J. Patel, S.K. Choi, Journal of Structural and Multidisciplinary Optimization.2012. 45(4), 529-543 (2012) https://doi.org/10.1007/s00158-011-

4. M. Jalalpour, M. Tootkaboni, Journal of Structural and Multidisciplinary Optimization, 53(4), 759-772 (2016)

5. L. Wang, D. Liu, Y. Yang, X. Wang, Z. Qiu, Applied Mechanics and Engineering, 326, 573-595 (2017)

6. S. Chakraborty, G. Bhattacharya, Proceedings of the International Symposium on Engineering under Uncertainty: Safety Assessment and Management (ISEUSAM 2012), Springer Science \& Business Media, Mar 12 (2013)

7. Z. C. Tang, Z. Z. Lu, D. W. Li, et al., Nonprobabilistic reliability analysis for an inside flap of an aircraft. J Aircraft, 49, 250-255 (2012)

8. M. Chiachio, J. Chiachio, G. Rus, Reliability in composites-A selective review and survey of current development, Composites Part B: Engineering (Elsevier, 2012)

9. Z.P. Qiu, R. Huang, X.J. Wang, W.C. Qi, Recent advances, Sci China-Phys Mech Astron September, 56(9) (2013)

10. E. Melchers, T. Andre, Structural Reliability Analysis and Prediction, 3rd Edition, ISBN: 978-1-119-26599-3 April 2018, 528 (2018)

11. O. Sigmund, K. Maute, Topology optimization approaches, Struct Multidiscip Optim, 48(6), 1031-1055 (2013)

12. L. Xia, P. Breitkopf, Matlab, Struct Multidiscip Optim, 52(6), 1229-1241 (2015)

13. L. Xia, Multiscale Structural Topology Optimization ISTE \& Elsevier, ISBN: 9781785481000, 184 (2016)

14. W. Zhang, J. Zhu, T. Gao, Topology Optimization in Engineering Structure Design /ISTE \& Elsevier, ISBN: 9781785482243, 294 (2016)

15. J.H. Zhu, W.H. Zhang, L. Xia, Topology optimization in aircraft and aerospace structures design, Arch Comput Methods Eng, 23(4), 595-622 (2016)

16. K. Guo, W.Z. Qi, B.T. Liu, et al. Optimization of an "area to point" heat conduction problem, Appl Therm Eng, 93, 61-71 (2016)

17. G. Kharmanda, I.R. Antypas, A.G. Dyachenko, International Journal of Mechnical Engineering Technology, 9(11), 810-820 (2018)

18. G. Kharmanda I.R. Antypas, A.G. Dyachenko, Journal of Mechnical Engineering Technology, 10(9), 1-11 (2019)

19. G. Kharmanda, I.R. Antypas, A.G. Dyachenko, E3S Web of Conferences 175(14) (2020)

20. G. Kharmanda, I. Antypas, A.G. Dyachenko, December 2020E3S Web of Conferences 210(4) (2020) 